\title{
Genes are quantum computers
}

\author{
Open Quantum Collaboration*† \\ July 22, 2020
}

\begin{abstract}
A sequence of logical arguments is presented in order to conclude that if biological cells are governed by quantum computational algorithms, then it is indeed possible to (re)program the genes' states in a quantum computer.
\end{abstract}

keywords: quantum biology, quantum computation, quantum biological computer

The most updated version of this paper is available at https://osf.io/a7tbu/download

\section{Introduction}

1. Quantum biology means that quantum theory can be applied to genes $[1-3]$.

2. Quantum computation is perhaps the most fundamental set of rules/ operations of nature $[4,5]$.

*All authors with their affiliations appear at the end of this paper.

†Corresponding author: mplobo@uft.edu.br | Join the Open Quantum Collaboration 


\section{Logic applied to quantum biology}

3. Humans are made by genes.

4. The genes are quantum biological entities.

5. Quantum mechanics operates in our genes.

6. Then we conclude that our genes are governed by quantum computational algorithms.

7. Therefore, genes can be simulated/deployed into a quantum computer.

\section{Final Remarks}

8. If our genes are ruled by quantum algorithms, then they can be fully (re)programmed as a quantum biological computer.

9. Gene reprogramming can be used to cure diseases [6].

\section{Open Invitation}

Review, add content, and co-author this paper $[7,8]$.

Join the Open Quantum Collaboration (https://bit.ly/ojmp-slack). Send your contribution to mplobo@uft.edu.br.

\section{Open Science}

The latex file for this paper together with other supplementary files are available [9]. 


\section{Ethical conduct of research}

This original work was pre-registered under the OSF Preprints [10], please cite it accordingly [11]. This will ensure that researches are conducted with integrity and intellectual honesty at all times and by all means.

\section{Acknowledgement}

+ Center for Open Science https://www.cos.io

+ Open Science Framework https://osf.io

\section{References}

[1] Lambert, Neill, et al. "Quantum biology." Nature Physics 9.1 (2013): $10-18$.

[2] Arndt, Markus, Thomas Juffmann, and Vlatko Vedral. "Quantum physics meets biology." HFSP Journal 3.6 (2009): 386-400.

[3] Vattay, Gabor, Stuart Kauffman, and Samuli Niiranen. "Quantum biology on the edge of quantum chaos." PloS one 9.3 (2014): e89017.

[4] Mermin, N. David. Quantum computer science: an introduction. Cambridge University Press, 2007.

[5] Nielsen, M. A., Chuang I. L. Quantum computation and quantum information. Cambridge University Press, 2010.

[6] Lobo, Matheus P. "Cancer: Division by Zero." OSF Preprints, 20 Jan. 2020. https://doi.org/10.31219/osf.io/34uft 
[7] Lobo, Matheus P. "Microarticles." OSF Preprints, 28 Oct. 2019. https://doi.org/10.31219/osf.io/ejrct

[8] Lobo, Matheus P. "Simple Guidelines for Authors: Open Journal of Mathematics and Physics." OSF Preprints, 15 Nov. 2019. https://doi.org/10.31219/osf.io/fk836

[9] Lobo, Matheus P. "Open Journal of Mathematics and Physics (OJMP)." OSF, 21 Apr. 2020. https://doi.org/10.17605/osf.io/6hzyp

[10] COS. Open Science Framework. https://osf.io

[11] Lobo, Matheus P. "Genes Are Quantum Computers." OSF Preprints, 20 June 2020. https://doi.org/10.31219/osf.io/a7tbu

\section{The Open Quantum Collaboration}

Matheus Pereira Lobo (lead author, mplobo@uft.edu.br) $)^{1,2}$

https://orcid.org/0000-0003-4554-1372

${ }^{1}$ Federal University of Tocantins (Brazil)

${ }^{2}$ Universidade Aberta (UAb, Portugal) 